\title{
Capítulo 2 Co-procesamiento en la industria del cemento
}

\section{Chapter 2 Co-processing in the cement industry}

FERRARESI-DE ARAUJO, Geraldo José ${ }^{\dagger}{ }^{*} \&$ NIÑO-CASTILLO, Jacob Elías ${ }^{2}$

${ }^{1}$ Universidade do São Paulo

${ }^{2}$ Universidad Autónoma de Guerrero

ID $1^{\text {er }}$ Autor: Geraldo José, Ferraresi-De Araujo / ORC ID: 0000-0002-2773-8641

ID $1{ }^{\mathrm{er}}$ Coautor: Jacob Elías, Niño-Castillo / ORC ID: 0000-0002-0575-5336, CVU CONACYT ID: 919977

DOI: $10.35429 / \mathrm{H} .2020 .2 .13 .23$

G. Ferraresi \& J. Niño 


\title{
Resumen
}

La industria del cemento consume mucha energía, a nivel mundial, el sector es responsable del 5-7\% de todas las emisiones de $\mathrm{CO}_{2}$ y aproximadamente el $3 \%$ de las emisiones totales de Gases de Efecto Invernadero (GEI). Por lo tanto, se debe prestar atención a la reducción de las emisiones de GEI relacionadas con la energía utilizada en el proceso, así como el co-procesamiento, una técnica en la que los residuos se utilizan para reemplazar las materias primas en la industria del cemento es de importancia ambiental estratégica. El objetivo principal es identificar las principales empresas en Brasil que utilizan el co-procesamiento y, como objetivo específico, identificar cómo las empresas que utilizan este proceso para generar una ventaja competitiva. El método fue una encuesta bibliográfica y se sometió a análisis de contenido. Votorantim y Lafargue Holcim ya han visto una ventaja competitiva del co-procesamiento, ya que reduce los costos y genera ingresos por la reutilización de los desechos.

\section{Industria del cemento, Coprocesamiento, Ventaja competitiva}

\begin{abstract}
The cement industry is energy intensive, globally the industry accounts for 5-7\% of all CO2 emissions and about $3 \%$ of total greenhouse gas (GHG) emissions. Therefore, focus should be given to the reduction of GHG emissions related to energy used in the process, so the coprocessing technique in which waste is used to replace raw materials in the cement industry is of strategic environmental importance. The main objective is to identify the main companies in Brazil that are using co-processing and, as a specific objective, identify how these companies are using this process to generate competitive advantage. The method was the bibliographical survey and submitted to content analysis. Votorantim and Lafargue Holcim have already seen competitive advantages from co-processing, as it reduces costs and generates revenues from the reuse of waste.
\end{abstract}

\section{Cement industry, Co-processing, Competitive advantage}

\section{Introducción}

En vista de las necesidades urgentes de reajustar los modelos de producción a escala global, ante las amenazas del calentamiento global y sus consecuencias, el co-procesamiento surge como una opción en línea con el desarrollo sostenible, ya que incluye las dimensiones ambientales, sociales y económicas de la sostenibilidad. Para Siddique y Naik (2004), debido a las crecientes preocupaciones sobre la conservación del medio ambiente y la calidad de vida de las generaciones futuras, el desarrollo económico, social y ambiental se ha convertido en el objetivo de muchas industrias.

El cemento, como el petróleo o el acero, es uno de los productos básicos vitales para el crecimiento de las economías. Ningún otro material es tan versátil como el cemento cuando se trata de construcciones de edificios, carreteras u obras de infraestructura importantes. El mercado mundial de cemento genera alrededor de \$250 mil millones/año de Dólares Americanos, sin contar a China, que representa más de la mitad de la producción y el consumo mundial, por lo que es abastecido prácticamente por empresas locales y, entre ellas, algunos de los mayores productores de cemento en el mundo, como el gigante estatal chino CNBM (China National Building Material), que es el mayor productor de cemento del mundo, con una capacidad instalada de 343 millones de toneladas de cemento por año, el frente suizo HOLCIM, cuya capacidad instalada en 2012 fue 218 Mt/año y el LAFARGE francés con 217 Mt/año (Cimento.org, 2013).

Específicamente con respecto al cemento, Huntzinger y Eatmon (2009) manifiestan que es un aglutinante hidráulico utilizado en la fabricación de mortero y concreto. Este producto representa uno de los materiales manufacturados más importantes del mundo. El cemento es el componente principal del hormigón, el material más consumido en la Tierra. Su producción alcanzó los 3600 millones de toneladas en 2012 y se estima que crecerá entre un 0.8 y un $1.2 \%$ anual lo cual representó entre 3700 y 4400 megatones. El cemento es un producto esencial, que proporciona a la sociedad lo que necesita en términos de viviendas seguras y cómodas e infraestructura moderna confiable. 
La producción de cemento implica el consumo de grandes cantidades de materias primas y combustibles, así como electricidad, aire y agua que involucra calentar una mezcla de piedra caliza, arcilla y bauxita a temperaturas entre $1200^{\circ} \mathrm{C}$ y $1500{ }^{\circ} \mathrm{C}$ (Lamas, et al, 2013). Este proceso proporciona la descomposición del carbonato de calcio en óxido de calcio, lo que provoca altas emisiones de $\mathrm{CO}_{2}$.

Además, para Jankovic, Walter, Eugene (2004), la producción requiere aproximadamente 110 $\mathrm{kWh} / \mathrm{t}$ de energía eléctrica, con un $40 \%$ dirigido a la molienda del clínker. Como industria intensiva en energía, generalmente es responsable del 50-60\% de los costos totales de producción. La energía térmica es responsable de aproximadamente el 20 al $25 \%$ del costo de producción de cemento. Esta energía se usa durante el proceso de combustión, mientras que la energía eléctrica se usa para moler cemento (Madlool, et al, 2013).

Este alto consumo de energía y la descarbonización de la piedra caliza hacen que la industria del cemento sea responsable de aproximadamente el 12 al $15 \%$ del uso total de energía industrial (Madlool, et al, 2011) y globalmente, el sector del cemento es responsable del 5-7 \% de todas las emisiones antropogénicas de dióxido de carbono y alrededor del $3 \%$ de las emisiones totales de GEI (Van Den Heede y De Belie, 2012).

Por ejemplo, cada tonelada de cemento Portland producido libera casi una tonelada de dióxido de carbono a la atmósfera, las emisiones globales de dióxido de carbono de la industria del cemento, que representan aproximadamente el $2.4 \%$ de las emisiones globales de $\mathrm{CO} 2$ de fuentes industriales y energéticas (Meyer, 2009).

Por lo tanto, la atención debe centrarse en reducir las emisiones de GEI relacionadas con la energía térmica y eléctrica utilizada en el proceso de producción, ya sea a escala local o global (GálvezMartos y Schoenberger, 2014).

Debido a esto, se hizo un esfuerzo significativo en términos de investigación y nuevos métodos desarrollados para lograr menores emisiones de $\mathrm{CO}_{2}$ dentro de la industria del cemento. Un método bien conocido y ampliamente utilizado es el co-procesamiento, una técnica en la que los desechos se utilizan para reemplazar las materias primas y/o combustibles (Cembureau, 2009).

Para Rocha, Lins y Santos (2011), el co-procesamiento en la industria del cemento es la mejor manera de recuperar energía y material de los residuos. Ofrece una solución segura y saludable para la sociedad, el medio ambiente y la industria del cemento al sustituir los recursos no renovables. El coprocesamiento de combustibles alternativos proporciona una solución en términos de reducir la dependencia de los combustibles fósiles, así como una contribución para reducir las emisiones.

El co-procesamiento, para Freitas y Nobrega (2014), es la recuperación simultánea de energía y el reciclaje de recursos minerales una vez que se utilizan combustibles alternativos, para reemplazar los combustibles fósiles primarios en hornos de cemento/clínker. Las fuentes tradicionales de energía en la fabricación de clínker de cemento son los combustibles fósiles como el carbón, el lignito, el coque de petróleo y el petróleo. En el co-procesamiento, estos son reemplazados por combustibles fósiles derivados de residuos y biomasa, en conjunto llamados combustibles alternativos y debido a las altas temperaturas y al tiempo de residencia, la destrucción de dichos combustibles derivados de residuos es eficiente y completa.

El co-procesamiento, según Cembureau (2016), de residuos en hornos de cemento, contribuye a la solución de tres problemas ambientales:

- $\quad$ Reducción del cambio climático: los combustibles alternativos son una de las principales palancas para la reducción de las emisiones de gases de efecto invernadero en la industria del cemento. Los combustibles alternativos pueden aportar 0.75 giga toneladas de $\mathrm{CO} 2$ en todo el mundo para reducir las emisiones de gases de efecto invernadero para 2050;

- Mejora de la gestión de residuos: el co-procesamiento de residuos puede reducir el volumen de residuos que se depositan en los vertederos y utilizar eficientemente su contenido energético; 
- $\quad$ Fomento de la economía circular: los minerales en los residuos se capturan en el producto de la planta de cemento y no necesitan ser depositados en vertederos, como suele ser el caso de la incineración de residuos.

- Los mayores productores de cemento en América Latina son Brasil, México y Argentina. Las compañías de cemento en estos países están en la lista de los 20 productores de cemento más grandes del mundo. Juntos, fueron responsables de la producción de 120 millones de toneladas de cemento en 2012 (Stafford, et al, 2015).

Países como Brasil, Colombia, Costa Rica, México y otros tienen estándares, regulaciones y leyes para el co-procesamiento y la gestión de residuos. Estas regulaciones varían según el país, pero, básicamente, prohíben el uso de desechos urbanos no tratados, desechos hospitalarios y desechos radiactivos, organoclorados y pesticidas (Idem, 2015).

En Brasil, la ley específica sobre co-procesamiento es la Resolución CONAMA No. 264, del 26 de agosto de 1999, publicada en el Boletín Oficial Federal No. 54, del 20 de marzo de 2000, que se aplica a la licencia de hornos de producción rotativos clínker para actividades de co-procesamiento de desechos, con la excepción de los desechos: desechos domésticos brutos, desechos de servicios de salud, radiactivos, explosivos, organoclorados, pesticidas y similares (Brasil, 1999), pero la falta de infraestructura para la gestión de residuos dificulta su práctica. De hecho, en 2007, 800000 toneladas de desechos industriales fueron coprocesadas en el país, pero corresponden solo al $30 \%$ de todos los desechos industriales producidos ese año. Los principales tipos de residuos utilizados son suelos contaminados, neumáticos, lodos aceitosos, catalizadores usados, adhesivos, resinas, látex, materiales recubiertos de goma y contaminados, como papel, plástico y madera (Ibídem, 2015).

Por lo tanto, dada la importancia social, económica y ambiental de la industria del cemento tanto en Brasil como en todo el mundo, se justifica un estudio sobre co-procesamiento, donde el objetivo principal del artículo es identificar las principales empresas en Brasil que están utilizando este proceso y como un objetivo específico para identificar cómo estas empresas que utilizan el co-procesamiento para generar una ventaja competitiva ecológica.

\section{Metodología}

Para lograr el objetivo general de la investigación, la metodología utilizada fue la investigación exploratoria, para Zikmund (2000), los estudios exploratorios diagnostican situaciones y exploran alternativas. Esta metodología se lleva a cabo durante la etapa inicial de un proceso de investigación donde se busca aclarar y definir la naturaleza de un problema.

La investigación se clasificó como cualitativa, ya que no se utilizaron datos estadísticos en este artículo. Con respecto a la recopilación de datos, el instrumento utilizado fue la revisión bibliográfica, según Lakatos y Marconi (2001), y cubre la literatura pública en relación con el tema estudiado, desde publicaciones científicas, disertaciones, revistas, libros, monografías, tesis y otros tipos de material donde el objetivo es poner al investigador en contacto con todo lo que se ha elaborado sobre el tema bajo investigación.

Con respecto al análisis de la información, el instrumento utilizado fue el análisis de contenido, según Bardin (1977), conceptualiza el análisis de contenido como: un conjunto de técnicas de análisis de comunicación con el objetivo de obtener, por procedimientos, una descripción sistemática y objetiva el contenido de los mensajes, indicadores, cuantitativos o no, que permiten la inferencia de conocimientos relacionados con las condiciones de producción y recepción de estos mensajes.

\section{Coprocesamiento: definición e importancia}

La gestión moderna de residuos debe incluir: i) eficiencia técnica en términos de protección ambiental, ii) eficiencia económica en términos de viabilidad de costos y iii) aceptabilidad social (Kikuchi y Gerardo, 2009). 
El co-procesamiento de residuos, según el World Business Council (2005), es un residuo seleccionado con valor calorífico recuperable que puede usarse como combustible en hornos de cemento al delegar una parte de los combustibles fósiles convencionales en el proceso de producción. Según la Asociación Europea de Cemento (CEMBUREAU), el co-procesamiento ofrece una solución en términos de reducir la dependencia de los combustibles fósiles, así como una contribución para reducir las emisiones de gases de efecto invernadero en la atmósfera.

El uso de residuos en el co-procesamiento de cemento tiene numerosos beneficios: menor necesidad de canteras, no tienen impactos negativos en las emisiones del proceso de producción, ni en la calidad ambiental y técnica del producto final; no afecta la salud y la seguridad de los trabajadores; destrucción completa de compuestos orgánicos; neutralización total de gases ácidos tales como óxidos de azufre y cloruro de hidrógeno; incorporación de trazas de metales pesados en la estructura del clínker con enlaces muy estables (formación de silicatos metálicos) y sin producción de subproductos como cenizas o residuos líquidos (Cembureau, 2009). Las características técnicas están de acuerdo con las directivas europeas, es decir, la directiva sobre incineración de residuos (Ibid, 2009).

Para Costa (2014), la designación Combustible Derivado de Residuos (CDR) (en inglés Combustible derivado rechazado o Combustible recuperado sólido) abarca una amplia gama de combustibles alternativos diferentes, ya que es posible producir combustibles basados en diferentes residuos. Para minimizar la generalización del término y regularizar la producción de CDR, la Comisión Europea publicó en 2006 la especificación CEN/TS 15357, que define el CDR como un combustible sólido preparado a base de residuos no peligrosos, producido con fines de recuperación de energía en plantas de incineración o co-incineración y que cumple con la clasificación y especificaciones presentadas en CEN/TS 15359.

El co-procesamiento de residuos en la industria del cemento proporciona un reemplazo máximo para materiales no renovables. La decisión sobre qué tipo de residuos finalmente se puede utilizar en una planta determinada no se responde de manera uniforme. Como regla básica, los desechos aceptados como combustible alternativo y/o materia prima deben agregar valor al horno de cemento en términos del valor calorífico de la parte orgánica y el valor material de la parte mineral (Cembureau, 2009).

La calidad de la CDR, de acuerdo con la especificación técnica CEN / TS 15359: 2006, se evalúa mediante el análisis de tres parámetros fundamentales, en los que se basa el sistema de clasificación: maximización de la eficiencia de la combustión térmica (parámetro PCI); bajo efecto de corrosión de los órganos de la caldera de combustión (parámetro $\mathrm{Cl}$ ) y bajos niveles de emisiones (parámetro $\mathrm{Hg}$ ).

En cuanto al sistema de clasificación, se basa en los valores límite de las tres propiedades, que se dividen en cinco clases. La clasificación se determina asignando una escala del 1 al 5 con respecto a cada parámetro, los valores límite para cada uno de los parámetros que clasifican un CDR se presentan en la siguiente Tabla 2.1 .

Tabla 2.1 Sistema de clasificación de residuos para co-procesamiento

\begin{tabular}{|l|l|l|r|r|r|r|r|}
\hline Parámetro & Medida Estadística & \multicolumn{5}{c|}{ Clases } \\
\hline PCI & Media & $\begin{array}{l}\text { MJ/kg } \\
\text { (tal como fue recibido) }\end{array}$ & $\geq 25$ & $\geq 20$ & $\geq 15$ & $\geq 10$ & $\geq 3$ \\
\hline Teor em Cl & Media & \% (base seca) & $\leq 0,2$ & $\leq 0,6$ & $\leq 1,0$ & $\leq 1,5$ & $\leq 3$ \\
\hline Teor em Hg & $\begin{array}{l}\text { Mediana } \\
\text { Percentil } 80\end{array}$ & $\begin{array}{l}\text { mg/MJ } \\
\text { (tal como fue recibido) mg/MJ } \\
\text { (tal como fue recibido) }\end{array}$ & $\leq 0,04$ & $\leq 0,03$ & $\leq 0,08$ & $\leq 0,15$ & $\leq 0,50$ \\
\cline { 3 - 8 } & & & & $\leq 0,06$ & $\leq 0,16$ & $\leq 0,30$ & $\leq 1,00$ \\
\hline
\end{tabular}

Fuente: Carvalho, 2011

Los principales componentes de los gases de escape de un horno de cemento son el nitrógeno del aire de combustión, la calcinación y el CO2 de combustión, el agua del proceso de combustión y las materias primas y el exceso de oxígeno. Los gases de escape también contienen pequeñas cantidades de polvo, cloruros, fluoruros, dióxido de azufre, NOx, dibenzo-p-dioxinas policloradas y dibenzofuranos policlorados (PCDD/PCDF) monóxido de carbono e incluso cantidades más pequeñas de compuestos orgánicos y metales pesados (Oak, 2011). 
El CDR no debe verse como un desperdicio, sino como un recurso o producto que tiene valor económico y ambiental, como parte de la cadena de valor de un sistema integrado de residuos. De ahí, que en América latina seguirá creciendo el sector de la construcción (Parra, 2020). Además, el CDR, al servir como un combustible alternativo al combustible fósil utilizado, permite no solo ahorros significativos en este combustible no renovable, sino también una reducción en las emisiones de CO2 y $\mathrm{CH} 4$, ganancias en créditos de carbono, espacio evitado en vertederos con reducción de costos operativos y economía de volumen ocupado con tarifa de gestión de residuos.

De acuerdo con Usón et. al. (2013), los desechos comunes utilizados en todo el mundo en las industrias del cemento son desechos urbanos sólidos, harina de carne y huesos de animales, lodos de depuradora, biomasa y neumáticos al final de su vida útil.

En América latina, los principales productores de cemento son Brasil, México y Argentina. En 2012 se encontraban entre los 20 principales productores de cemento del mundo, ocupando el quinto, el duodécimo y el décimo octavo, respectivamente (Stafford, et al, 2015). Por lo tanto, las reglas sobre qué residuos se pueden usar y los límites para la emisión a la atmósfera de contaminantes deben estar bien definidos y estrictamente cumplidos e inspeccionados, por lo tanto, se han hecho muchos esfuerzos en América latina.

Pero mientras los países latinoamericanos están desarrollando leyes y estrategias con respecto al co-procesamiento de residuos industriales, muchos países europeos están co-procesando no solo los residuos industriales, sino también los residuos urbanos (Usón, et al, 2013). Ello, también coincide con lo expuesto actualmente en la transformación ecológica “...deben existir una implementación de gestión sostenible que controle desde la fabricación de productos que se puedan reutilizar y reciclar, generando una economía circular...” (Tinjacá, Ruiz y Alfonso, 2019: 10).

\section{Ventajas competitivas ecológicas y las principales empresas cementeras que utilizan el coprocesamiento}

Las ganancias ambientales discutidas anteriormente están en línea con las buenas prácticas ambientales, tales herramientas generan una ventaja competitiva a través de estrategias de bajo costo o diferenciación de acuerdo con las dimensiones de competitividad en las que se posiciona cada organización (Porter, 1980). La administración de materias primas, de acuerdo con Brito y Beraldi (2010), de manera eficiente y efectiva es fundamental para la reducción de pérdidas en el proceso de producción, por lo tanto, son fuentes de reducción de costos. Dichas prácticas pueden llevar a las compañías a lograr una diferenciación a escala global, donde las restricciones ambientales están aumentando como sucede en Colombia, Argentina, México y Brasil (Barrios y Pérez, 2019).

El cuidado ambiental se vincula con la calidad en la medida que ofrece ventajas competitivas en dos maneras: la primera, en el corto plazo implica ofrecer en la industria cementera cero residuos y contaminantes a la atmósfera lo que permite incrementar los ingresos año con año en la empresa Holcim, en tanto que, la segunda hace alusión a la calidad mejorada de sus productos, de tal manera que, es el camino más efectivo para que una empresa privada como la que se analiza florezca en cualquier parte del orbe (Niño, Niño y Niño, 2017).

Además, la presión reguladora de parte de los gobiernos, así como de una parte de la sociedad, también es un estímulo para la innovación, ya sea en productos o procesos, ya que los desafíos globales de escasez e inflación de los recursos naturales inevitablemente limitarán la actividad económica. La capacidad de las organizaciones para hacer frente a tales restricciones conduce a un modelo de gestión ambiental que abarca desde la prevención de la contaminación hasta el desarrollo sostenible, a través del meticuloso proceso de conocimiento de toda la cadena (Brito y Beraldi, 2010). Dentro del contexto antes mencionado, vale la pena mencionar Votorantim Cimentos y Lafargue Holcim con énfasis en el mercado nacional de cemento.

Votorantim Cimentos es una de las diez compañías de concreto y agregados más grandes del mundo y actualmente es el líder en el mercado nacional de materiales básicos para la construcción civil. Actualmente, cuenta con 18 plantas de cemento en Brasil, cuatro unidades de agregados, cuatro unidades de productos complementarios (mortero, cal y piedra caliza agrícola), 105 plantas de concreto y 60 centros de distribución. 
En los Estados Unidos y Canadá, cuenta con siete unidades de producción de cemento, 150 plantas de concreto, seis barcos y 12 terminales de cemento en la región de los Grandes Lagos. En Bolivia, hay un molino ubicado en Puerto Suárez. Aunado a lo anterior, tiene una cartera de más de 25 mil clientes directos y produce más de 40 artículos en los segmentos de cemento, mortero, lechada, yeso, cal, caliza agrícola, concreto y agregados (Votorantim Cimentos, 2018).

Además, según Votorantim Cimentos (2018), la compañía fue la primera en Brasil en utilizar el co-procesamiento en su proceso de fabricación de cemento, donde el total de residuos coprocesados en las unidades de la compañía supera las 600 mil toneladas por año.

Actualmente, la compañía aplica el co-procesamiento a 15 de sus unidades operativas y planea invertir más de R \$ 300 millones en co-procesamiento para 2020, con la intención de alcanzar el 30\% del uso de residuos para quemar clínker en todas sus plantas en el país y alcanzar niveles internacionales en el uso de combustibles y materias primas renovables en las operaciones nacionales de cemento. Además, el proyecto implicará el uso de una variedad de caña de azúcar para alimentar el horno de cemento, cerca de la producción de carbono neutral. En Brasil, el 25\% de todo el combustible usado era renovable (Revista Amazonia, 2017).

Con respecto a Lafarge Holcim, es el líder mundial en materiales y soluciones para la construcción que presta servicios en todo el mundo, donde la compañía tiene alrededor de 90 mil empleados en más de 80 países y tiene una presencia equilibrada entre los mercados en desarrollo y maduros. Específicamente en Brasil, con ventas brutas de R \$873 millones y más de 1600 empleados (Holcim Brasil S. A.), es el cuarto mayor fabricante de cemento en el país y también uno de los líderes en el suministro de concreto y agregados. La empresa forma parte del grupo suizo Holcim Ltd., su gran diferencial es proporcionar soluciones integradas en cemento, hormigón y áridos, ofrece asesoramiento técnico y alta tecnología. Holcim fue la primera industria del cemento en el país en obtener la certificación ISO 9000. Sus inversiones en el medio ambiente también le garantizaron el estatus de pionero en el sector al obtener ISO 14000 (Lafarge Holcim, 2018 y Ferraresi de Araujo, 2020).

Con respecto al co-procesamiento, según eCycle (2017) Lafargue Holcim tiene una unidad comercial estratégica, Geocycle, responsable de la gestión de los residuos industriales y urbanos que se utilizan como combustibles alternativos o sustitutos de las materias primas en los hornos cemento de coprocesamiento. Esta tecnología ya se utiliza en las unidades de producción de Holcim en Cantagalo-RJ, Barroso-MG y Pedro Leopoldo-MG, donde estas unidades trituran y mezclan residuos industriales líquidos, sólidos y pastosos.

Los principales residuos recibidos por Holcim son: sustancias oleosas; catalizadores usados; resinas, pegamentos y látex; neumáticos y caucho; disolventes lías ácidas; lodos de tratamiento; tierra contaminada; revestimientos de aluminio para tanques; papel y cartón contaminados; y residuos no peligrosos, como neumáticos, plástico, madera y aguas residuales, de modo que tengan un valor calorífico de al menos $2000 \mathrm{kcal} / \mathrm{kg}$. En todo el mundo, alrededor del $80 \%$ de los materiales coprocesados por Holcim son aceites usados y desechos no peligrosos, como los mencionados anteriormente. En Brasil, como en otras unidades en todo el mundo, aproximadamente el $80 \%$ de los desechos se utilizan como sustituto de combustible y el $20 \%$ como materia prima (Furtado, 2013).

\section{Consideraciones finales}

La metodología exploratoria permitió aclarar que el co-procesmiento en la industria del cemento tiene efectos positivos en cuanto a la dimensión ambiental debido a que la tecnología actual contribuye a la reducción de desechos como tierra contaminada, neumáticos, lodos aceitosos, catalizadores usados, adhesivos, resinas, látex, materiales recubiertos de goma y contaminados, como papel, plástico y madera desechados de manera inapropiada en el medio ambiente. Al mismo tiempo, el consumo de combustibles fósiles disminuye en el proceso de fabricación del cemento. Además, no representa ningún riesgo para las comunidades que rodean las plantas de cemento. 
Por lo tanto, el co-procesamiento en la industria del cemento, tanto en Brasil como en todo el mundo, es una cuestión no solo de cumplimiento de las normas ambientales, como DN COPAM 26 y la Resolución CONAMA 264, sino también una fuente de ventaja competitiva ecológica, donde la generación de valor de estas organizaciones para la sociedad es mayor que el precio final de sus productos a sus clientes. En consecuencia, cuanto mayor sean los impactos resultantes del empeoramiento del problema ambiental, mayor será el valor de estas empresas entregadas a sus clientes, y mayor será la presión por parte del gobierno y la sociedad de aquellos que no son adecuados para los nuevos estándares de producción.

La revisión bibliográfica permitió observar que, en la dimensión social, el co-procesamiento en la industria del cemento contribuye a la generación de empleos, así como la necesidad de que los recursos humanos involucrados en este proceso realicen la separación de los residuos que se utilizarán en el coprocesamiento, además de su vital intervención en la logística de estos residuos a los centros de productos de cemento. Aunque los beneficios del co-procesamiento son sustanciales, la demora en la concesión de licencias para este tipo de servicio ambiental sigue siendo un desafío en Brasil, ya sea debido a la falta de profesionales involucrados, así como a la demora en el proceso de vinculación con instituciones públicas.

El análisis de contenido aplicado hace alusión a la importancia del co-procesamiento en la industria del cemento en la dimensión económica, debido a que contribuye financieramente a la empresa cementera privada en la medida en que el co-procesamiento es un servicio-pago, por lo que los generadores de residuos pagan por su eliminación; de lo contrario, las empresas cementeras pueden reducir el costo de comprar energía y combustibles en el proceso de producción sin perder eficiencia energética. De tal forma que, empresas como Votorantim Cimentos y Lafargue Holcim ya se han dado cuenta de la importancia de utilizar el co-procesamiento como una forma de ventaja competitiva ecológica, ya que los interesados (proveedores, clientes, accionistas, empleados, comunidad, sociedad y alta gerencia) están cada uno más preocupados por los problemas ambientales, sin perder la racionalización de los costos del proceso de producción.

\section{Referencias}

Bardin, L. (1977). Análise de conteúdo. Lisboa: Edições 70.

Barrios, R. y Pérez, G. A. (2019). Análisis del contexto normativo del contrato en Colombia y legislaciones Argentina, México y Brasil. Tesis de Licenciatura. Facultad de Derecho. Politécnico Gran Colombiano. http://alejandria.poligran.edu.co/handle/10823/1507

Brasil. (2000). Diário Oficial da União, Brasília. Resolução CONAMA Núm. 264, de 26 de agosto de 1999. Licenciamento de fornos rotativos de produção de clínquer para atividades de co-processamento de resíduos.

Brito, R. P. y Berardi, P. C. (2010). Vantagem competitiva na gestão sustentável da cadeia de suprimentos: um meta estudo. Revista Administração de Empresas, 50(2), 155-169. Disponible en http://www.scielo.br/scielo.php?script=sci_arttext\&pid=S0034-

$75902010000200003 \& \operatorname{lng}=$ en\&nrm=iso

Carvalho, I. R. G. (2016). CDR um resíduo ou um produto, e a sua viabilidade técnico-económica: análise do estudo do caso. 2011. Dissertação (Mestrado em Engenharia Ambiental). Faculdade de Ciências e Tecnologia, Universidade de Nova Lisboa, Lisboa. 2016. Disponible en https://run.unl.pt/bitstream/10362/5941/1/Carvalho_2011.pdf

Cembureau. (2016). Market opportunities for use of alternative fuels in cement plants across the EU. Disponible en https://cembureau.eu/media/1231/ecofysreport_wastetoenergy_2016-07-08.pdf

Cemboreau (2009). Sustainable cement production: Co processing of alternative fuels and raw materials in the European industry. 2009. Disponible en www.cembureau.eu

Cimento.org. (2013). A indústria do cimento. Disponible en: https://cimento.org/cimento-no-mundo2013/ 
Costa, J. M. B. (2014). Coprocessamento de CDR no processo de produção de cimento. Dissertação (Mestrado em engenharia química). Faculdade de Ciências e Engenharia do Ambiente. Universidade de Nova Lisboa, Lisboa, 2014. Disponible en: https://run.unl.pt/bitstream/10362/15783/1/Costa_2014.pdf

Ecycle. (2017). O que é coprocessamento e quais as vantagens ambientais que ele pode oferecer?. Disponible en https://www.ecycle.com.br/component/content/article/67-dia-a-dia/5918-o-que-ecoprocessamento-e-quais-as-vantagens-ambientais-que-ele-pode-oferecer-.html

Ferraresi de Araujo, G. J. (2020). O coprocessamento na industria de cimento: definicao, oportunidades e ventagem competitiva. Revista Nacional de Gerenciamento de Cidades. 8(57), 53-61. https://d1wqtxts1xzle7.cloudfront.net/62707296/O_coprocessamento_na_industria_de_cimento_definic ao_oportunidades_e_vantagem_competitiva20200401-47892-ks7tms.pdf?1585801105=\&responsecontent-

disposition=inline\%3B+filename\%3DO_coprocessamento_na_industria_de_ciment.pdf\&Expires=1596 927663\&Signature=JuTxkrphQllt $\sim$ K8I6gAdH2nWLipDApCchKwR-UknxlXMaw47BGjStBifyZeO-dxKrADSrbsOMowY0jUygPIqRt0PrGK67ZX13lepnnBTCIRv1RO3fV8wW0w2GZ-

afgj31LzmBT9oit1EPtcg9bIMtnspTaN5JfN0aQsqPlquFq359fTpjQI0mAwxIpdUTFmnoKpeTYNwzuz DGyiFzCp6BHlOog4g3uhT6EqjIy yRMMymPnC-cUM $\sim 10 p i i B j 12 g D 5 g 0 G n X Q-$ mF33fh5XhuJiL8L41DdX7r9OEzhIxciUELcNxaySTTjmiAlNLdDi4upQUGZ2IovAnNZCKInxRJ0g _\&Key-Pair-Id=APKAJLOHF5GGSLRBV4ZA

Freitas, S. S. y Nobrega, C. C. (2014). Os benefícios do co processamento de pneus inservíveis para a indústria cimenteira. Engenharia Sanitaria e Ambiental, 19(3), 293-300. Disponible en http://www.scielo.br/scielo.php?script=sci_arttext\&pid=S1413-

41522014000300293\&lng=en\&nrm=iso. DOI: 10.1590/S1413-41522014019000000769

Furtado, M. (2013). Tecnologia ambiental co processamento: Uso de resíduos em forno de cimento cresce 25\%. Química.com.br, São Paulo, 2013. Disponible en https://www.quimica.com.br/tecnologiaambiental-coprocessamento-residuos-fornos-cimento-cresce-25/

Galvez-Martos, J. L. y Schoenberger, H. (2014). An analysis of the use of life cycle assessment for waste co-incineration in cement kilns. Resources, Conservation and Recycling, 88(1), 118-131. Disponible en https://www.sciencedirect.com/science/article/pii/S0921344914000548/.

10.1016/j.resconrec.2014.02.009

Huntzinger, D. N. y Eatmon, T. D. (2009). A life-cycle assessment of Portland cement manufacturing: comparing the traditional process with alternative technologies. Journal of Cleaner Production, 17(7), 668-675. Disponible en https://www.sciencedirect.com/science/article/pii/S0959652608000826. DOI: https://doi.org/10.1016/j.jclepro.2008.04.007

Jankovic, A.; Walter, V. y Eugene, D. (2004). Cement grinding optimisation. Minerals Engineering, 17(1), 1075-81. Disponible en http://citeseerx.ist.psu.edu/viewdoc/download?doi=10.1.1.91.6206\&rep=rep1\&type=pdf. $\quad$ DOI: https://doi.org/10.1016/j.mineng.2004.06.031

Kikuchi, R. y Gerardo, R. (2009). More than a decade of conflict between hazardous waste management and public resistance: A case study of NIMBY syndrome in Souselas (Portugal). Journal of Hazardous Materials, 172(2-3), 1681-1685. Disponible https://www.sciencedirect.com/science/article/pii/S0304389409011765. DOI: https://doi.org/10.1016/j.jhazmat.2009.07.062

Lafarge Holcim (2018). Quem somos. Disponible en https://www.lafargeholcim.com.br/quem-somos

Lakatos, E. M. y Marconi, M. A. (2001). Fundamentos metodologia científica. São Paulo: Atlas.

Lamas, W. D. Q.; Palau, J. C. F. y Camatgo, J. R. (2013). Waste materials co-processing in cement industry: Ecological efficiency of waste reuse. Renewable and Sustainable Energy Reviews, 19(1), 200207. Disponible en https://www.sciencedirect.com/science/article/pii/S1364032112006260. DOI: https://doi.org/10.1016/j.rser.2012.11.015 
Madlool, N. A.; Rahman, S.; Hossain, M.S. y Rahin, N.A. (2011). A critical review on energy use and savings in the cement industries. Renewable and Sustainable Energy Reviews, 15(4), 2042-2060. Disponible en https://www.sciencedirect.com/science/article/pii/S1364032111000207. DOI: 10.1016/j.rser.2011.01.005

Madlool, N. A.; Saidur, R.; Rahin, N. A. y Kamalisarvestani, M. (2013). An overview of energy savings measures for cement industries. Renewable and Sustainable Energy Reviews, 19(1), 18-29. Disponible en https://www.sciencedirect.com/science/article/pii/S1364032112005977. DOI: https://doi.org/10.1016/j.rser.2012.10.046

Niño, N., Niño, I, y Niño, J. (2017). Ventaja competitiva de la UAGro: por área de investigación CONACYT. En Sánchez, J. y Mayorga, P. I. (Coords). El valor del conocimiento y efectos en la competitividad. Guadalajara: RIICO. https://www.riico.net/index.php/riico/article/view/1470

Parra, C. A. (2020). Estrategias de Responsabilidad Social y Ambiental de la Empresa Holcim en México. Tesis de Maestría. Universidad del Rosario. Colombia. https://repository.urosario.edu.co/handle/10336/24366

Porter, M. E. (1980). Competitive strategy: techniques for analysing industries and competitors. New York: Free Press.

Revista Amazonia. (2017). Votorantim Cimentos investiu R\$ 136 milhões em coprocessamento de resíduos nos últimos 4 anos: Modernização de equipamentos e novas tecnologias contribuem de forma sustentável para maior redução de emissões na produção de cimento. Disponible en https://revistaamazonia.com.br/votorantim-cimentos-investiu-136-milhoes-coprocessamento-residuosnos-ultimos-anos/

Rocha, S. D. F.; Lins, V. F. C. y SANTO, B. C. E. (2011). Aspectos do coprocessamento de resíduos em fornos de clínquer. Engenharia Sanitária e Ambiental, 16(1), 1-10. Disponible en http://www.scielo.br/pdf/esa/v16n1/a03v16n1. DOI: http://dx.doi.org/10.1590/S141341522011000100003

Siddique, R. y Naik, T. R. (2004). Properties of concrete containing scrap-tire rubber-an overview. Waste Management, 24(6), 563-569. Disponible https://www.sciencedirect.com/science/article/pii/S0956053X04000212. DOI: 10.1016/j.wasman.2004.01.006

Stafford, F. N.; Viquez, M. D.; Labrincha, J. y Hotza, D. (2015). Advances and challenges for the coprocessing in Latin American cement industry. Procedia Materials Science, 9(1), 571-577. Disponible en https://www.sciencedirect.com/science/article/pii/S2211812815002163. DOI: https://doi.org/10.1016/j.mspro.2015.05.032

Tinjacá, A. M., Ruiz, Y. A. y Alfonso, D. F. (2019). C. I. Recyclables S. A. S. una mirada hacia la sostenibilidad. Facultad de Ciencias Económicas y Administrativas. Universidad Agustiniana. http://repositorio.uniagustiniana.edu.co/handle/123456789/1099

Usón, A. A. y Ferreira, G. A. (2013). Uses of alternative fuels and raw materials in the cement industry as sustainable waste management options. Renewable and Sustainable Energy Reviews, 23(1), 242-260. Disponible en https://www.sciencedirect.com/science/article/pii/S1364032113001263. DOI: 10.1016/j.rser.2013.02.024

Van Den Heede, P. y De Belie, N. (2012). Environmental impact and life cycle assessment (LCA) of traditional and 'green'concretes: literature review and theoretical calculations. Cement and Concrete Composites, 34(4), 431-442. Disponible https://www.sciencedirect.com/science/article/pii/S0958946512000054. DOI: 10.1016/j.cemconcomp.2012.01.004 
Votorantim Cimentos. (2016). Votorantim Cimentos apresenta novo portfólio: a maior inovação em 83 anos de história. Disponible en https://www.votorantimcimentos.com.br/noticia/votorantim-cimentosapresenta-novo-portfolio-maior-inovacao-em-83-anos-de-historia/

World Business Council for Sustainable Development. (2005). Guidelines for the selection and use of fuels and raw materials in the cement manufacturing industry. In: Cement sustainability initiative (CSI). 2005. Disponible en http://wbcsdpublications.org/project/guidelines-for-the-selection-and-use-of-fuelsand-raw-materials-in-the-cement-manufacturing-process/

Zikmund, W. G. (2000). Business research methods. Fort Worth, TX: Dryden. 